\title{
Astronomical Tests for Extended Gravity: Possible Constraints on $f(R)$ with Vanishing Cosmological Constant
}

\author{
Stanislav Alexeyev ${ }^{1, \star}$, Boris Latosh ${ }^{2,3}$, and Vsevolod Echeistov ${ }^{4}$ \\ ${ }^{1}$ Sternberg Astronomical Institute, Lomonosov Moscow State University, Universitetsky Prospekt, 13, \\ Moscow 119234, Russia \\ ${ }^{2}$ Department of Theoretical Physics, Faculty of Natural and Engineering Science, Dubna International Uni- \\ versity, Universitetskaya Str., 19, Dubna, Russia \\ ${ }^{3}$ Bogoliubov Laboratory of Theoretical Physics, Joint Institute for Nuclear Research, Joliot-Curie 6, 141980 \\ Dubna, Russia \\ ${ }^{4}$ Department of Astrophysics and Stellar Astronomy, Physics Faculty, Lomonosov Moscow State University, \\ Vorobievi Gori, 2/1, Moscow 119234, Russia
}

\begin{abstract}
We discuss the predictions of Starobinsky model of $f(R)$-gravity with vanishing cosmological constant at the scales, typical for galaxies and galaxy clusters. We show that at current observational accuracy there is no significant difference in cut-off radius values for Starobinsky model and general relativity. This occurs from the masses of galaxies (from $10^{9} M_{\text {Sun }}$ ), till galaxy clusters ones (till $10^{18} M_{\text {Sun }}$ ). In addition we demonstrate that cut-off radius value dependence against Starobinsky model parameter $n$ increases during increasing $n$ for all mass range. Despite the fact that some models provide the cutoff radius value less than in general relativity no any model comes outside the existing observational accuracy frames.
\end{abstract}

\section{Introduction}

The general relativity (GR) is widely treated as main theory of gravitation. It successfully describes all observable gravitational phenomena on the scales of the solar system [1]. The recent discovery of gravitational waves [2] confirmed the applicability of GR to describe the gravitational interaction [3] over a wide range of distances. Nevertheless, there is reason to believe that GR is not able to provide a fully correct description of gravity on large scales and in the regime of huge energies. Indication of the incomplete astrophysical applicability of GR is the Universe accelerated expansion [4]. This phenomenon is described by a cosmological constant most accurately. As a consequence, the introduction of an additional constant term to the action causes difficulties in matching with the quantum theory $[5,6]$. The incomplete applicability of GR in the high-energy regime is indicated by several phenomena. First, GR is not renormalizable [7], therefore, in the quantum regime, it could be treated only as an effective field theory [8]. Further, the construction of self-consistent cosmological models requires the introduction of an inflationary stage, which cannot be obtained directly from GR [9]. To try to solve this problem the gravity models were extended by additional

^e-mail: alexeyev@sai.msu.ru 
fields and curvature expansions [10-12]. Here we discuss of $f(R)$-gravity (the Lagrangian is a function $f$ of scalar curvature $R$ [13]). The choice of function $f$ is not arbitrary, otherwise instabilities may appear $[14,15]$. Lagrangians of such type arise during taking into account the radiative corrections of quantum field theory in curved space-time [16], and also in string theories [17].

Further, the Newtonian theory of gravitation can be treated as asymptotes of a more general theory of gravity (GR) in weak gravitational fields. Following this logic, we can assume that GR itself is an approximation on the scales of the solar system of a more general theory. Such approach allows considering the extended theories of gravity of various kinds necessary for the description of dark matter and dark energy. For an explanation of dark matter and dark energy problems are offered, on the one hand, the undiscovered population of objects of known types (black holes and other objects) and new types of particles and fields ( " new physics"), on the other is extended theory of gravity $[12,18]$ (" new gravity"). The combination of these approaches makes possible to use the strengths of each of them. For example, in the framework of the scalar-tensor gravity [10].

The problem of dark energy and, consequently, the cosmological constant, is a strong stimulus for the modification of GR. Some models treat dark energy as the effect of the space-time geometry: massive gravity, where the gravitational interaction carrier (graviton) itself has a nonzero mass, theories with violation of Lorentz invariance (under extreme conditions), or, theories with additional fields or space-time dimensions (which can also be represented as additional fields) [10, 19]. Taking into account the relationship between $f(R)$ gravity and scalar-tensor models, their mutual transformations [20], as a first step we discuss the model proposed in [15]. It is constructed to satisfy two requirements. The function $f(R)$ must be regular and becomes equal to zero for $R=0$. Models in which the function $f$ diverges in the region of small curvature are indistinguishable from GR or do not provide an adequate description of the existing observational data (for a detailed discussion, see [15]). In addition, the scale of the curvature $\Lambda$ is introduced to the model and is interpreted as the magnitude of the effective cosmological constant. For $R \gg \Lambda$, the function $f$ tends to $R-2 \Lambda$. Here the effective cosmological constant disappear in the low curvature regime, therefore models of such class are called " models with a vanishing cosmological constant". In the framework of this paper, for the sake of simplicity, we refer to the model proposed in [15] as "Starobinsky model".

In [15] the following form of the function $f$ is suggested:

$$
f(R)=R+\lambda R_{0}\left(\left(1+\frac{R^{2}}{R_{0}^{2}}\right)^{-n}-1\right),
$$

where $R$ is the scalar curvature, $\lambda, R_{0}$ and $n$ are the model parameters. It is assumed that $\lambda>0$, and $R_{0}$ coincides with the cosmological constant in order of magnitude. As it was shown in [15] for large values of curvature (effective) the cosmological constant is expressed in terms of the parameters as:

$$
\Lambda=\frac{\lambda}{2} R_{0}
$$

Therefore for convenience we suppose that $\lambda=2$ and $R_{0}=\Lambda$.

According to [15], in the intermediate curvature mode, when $0<R<\Lambda$, the Starobinsky model differs from GR and can describe the effects of dark matter. Laboratory tests and observations in the solar system provide the following limitation: $n \geqslant 2$. The model should describe gravitational phenomena on all scales; therefore we study its manifestations on the scales of galaxies and clusters of galaxies.

In order to verify the model, it is necessary to use values associated with real observational data. It is useful to address the effects in gravitationally coupled systems. At this level, the Universe expansion manifests itself as a force acting equally on all bodies and preventing their gravitational connection. 
The balance of the force of gravitational attraction and the force associated with the accelerated expansion defines a specific scale. This manifold is called as "the turning radius". It determines the maximum size of the gravitational bound system [21, 22].

Thus, it is possible to compare the turning radii predicted by GR and Starobinsky model and with the observed spatial scales of galaxies and galaxy clusters. Such an activity could make possible to establish additional restrictions on the parameters of the Starobinsky model on these scales.

\section{Turning radius calculation}

The spheroidal and some elliptical galaxies, as well as the so-called regular clusters of galaxies, have a spherical or close to it shape, small rotational velocities (in comparison with the dispersion of velocities), and a pronounced central part of [23-25]. Therefore, to describe the gravitational field of the galaxy, one can use a spherically symmetric metric, assuming that the most part of the mass is concentrated in the center. So, we can use the Schwarzschild type of the metric, which we represent in the form:

$$
d s^{2}=e^{A} d t^{2}-e^{-A} d r^{2}-r^{2} d \Omega,
$$

where $A=A(r)$ is unknown function.

Field equations in $f(R)$-gravity have the following form $[13,15]$ :

$$
f^{\prime}(R) R_{\mu \nu}-\frac{1}{2} f(R) g_{\mu \nu}-\left(\nabla_{\mu} \nabla_{v}-g_{\mu \nu} \square\right) f^{\prime}(R)=0,
$$

where $f^{\prime}(R)=d f(R) / d R, \nabla_{\mu}$ is covariant divergence, $\square=\nabla_{\mu} \nabla^{\mu}$ is d'Alambert operator. Eqs ((4)) represent nonlinear system of second order, which could be solved numerically. To simplify we descrease the equations order by the introducing a new variable $a$ :

$$
a=\frac{d A}{d r} .
$$

Similarly, for simplicity, we assume the scalar curvature as the independent variable. As an equation connecting it with $A$ and $a$ we use its explicit form:

$$
R=-\frac{2}{r^{2}}+e^{A}\left(\frac{d a}{d r}+a^{2}+\frac{4 a}{r}+\frac{2}{r^{2}}\right) .
$$

From $(1,1)$-component of eqs $((4))$ it is possible to rewrite $d^{2} f / d r^{2}$ and using $(0,0)$-component of $((4))$, to show that:

$$
\frac{d R}{d r}=\frac{f^{\prime}(R) \cdot R_{00} / g_{00}-f(R) / 2}{\left(e^{A}(a+2 / r)+a / 2\right) f^{\prime \prime}(R)} .
$$

So, we consider the equations $((5)),((6))$ and (7) together:

$$
\left\{\begin{array}{l}
\frac{d A}{d r}=a, \\
\frac{d a}{d r}=e^{-A}\left(R+\frac{2}{r^{2}}\right)-a^{2}-\frac{4 a}{r}-\frac{2}{r^{2}}, \\
\frac{d R}{d r}=\frac{f^{\prime}(R) \cdot R_{00} / g_{00}-f(R) / 2}{\left(e^{A}(a+2 / r)+a / 2\right) f^{\prime \prime}(R)},
\end{array}\right.
$$

where $f$ is defined in ((1)) and Ricci tensor components are calculated using the metrics ((3)). 
The system ((8)) is solved with the modified Runge-Kutta method (TR-BDF2, [26]). The mass spectrum is chosen from $10^{9} M_{S \text { un }}$ to $10^{18} M_{S \text { un }}$ because these values that are typical for individual galaxies (Mass of the Milky Way Galaxy -4 times $10^{11} M_{\text {Sun }}$ [27]) and for clusters (the characteristic masses of the clusters are $\sim 10^{15} M_{S \text { un }}[28-30]$ ) respectively. We checked the values of the parameter $n$ from 1 to 9 using the following definition of the gravitational potential:

$$
\phi=\frac{c^{2}}{2}\left(g_{00}-1\right)=\frac{c^{2}}{2}\left(e^{A}-1\right),
$$

as well as its dimensionless version:

$$
\phi / c^{2}=\frac{e^{A}-1}{2} .
$$

The turning radius, as indicated above, is located at the point where the force of gravitational attraction is balanced by the one associated with the Universe accelerated expansion. At this point the gravitational potential must vanish:

$$
\frac{d \phi}{d r}=0
$$

Since $a$ is an independent variable, one can look for $r_{1}$ and $r_{2}$ for which $a\left(r_{1}\right)>0$ and $a\left(r_{2}\right)<0$, respectively. Further it is easy to calculate the desired value of the turning radius using the linear interpolation. The dependence of the radius against the mass of the object (a galaxy or a cluster of galaxies) for Starobinsky's models with various $n$ values and GR is shown in Fig. 1. The dependencies are very close to the power law, so, on a logarithmic scale, they look linear. We approximate the dependence of the radius $R_{T A R}$ of the turning one by the following form:

$$
R_{T A R}=M^{\alpha} \times R_{T A R}\left(M_{S o l}\right),
$$

Where $R_{T A R}$ is expressed in kiloparsecs $(k p c)$, and the mass of the object $M$ is expressed in the masses of the Sun $\left(M_{S \text { un }}\right)$. In this logarithmic form:

$$
\log _{10}\left(R_{T A R}\right)=\alpha \log _{10}(M)+\beta
$$

We illustrate the values of the ratio of the radii of the rotation of the Starobinsky model and GR against the mass graphically in Fig. 2. In the whole range of considered masses, the Starobinsky models with $n=1$ and $n=2$ have the least radii of zero mass, and therefore they should be compared with observations first of all.

\section{Comparing with observational data}

Direct measurements of the turning radius at this stage of tool development are unreal. Really it is necessary to observe directly the motion of a test body, for example, a star in a distant galaxy. Such observations are still beyond modern possibilities. But, taking the density profile obtained from gravitational lensing one can use it as a down limit of the size of the object. The turning radius would limit the maximum size of the object. It cannot be less than the size of the density profile. Similar profiles were obtained from the analysis of CLASH [28, 29] and other clusters of galaxies [30]. All these data are comparable with $n=1$ and $n=2$ models of Starobinsky, which have the smallest value of the turning radius, and also with GR.

Despite significant differences, none of the models discussed (even with $n=1$ that does not pass the solar system tests [15]) does not deviate further than by $20 \%$ from the radius determined by GR and fixed by the observational data. Thus, the current level of accuracy of astronomical observations does not allow strengthening or confirming the earlier limitations on the Starobinsky model. 


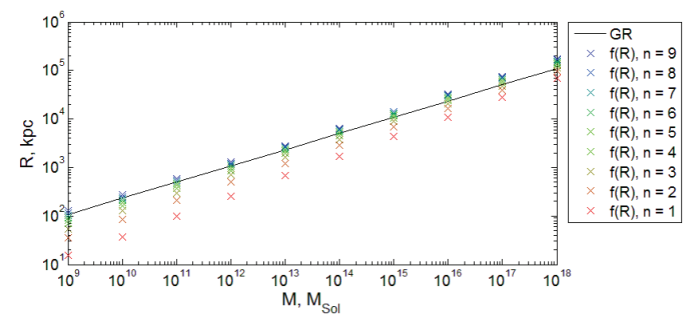

Figure 1. Dependence of the radius against the mass for the Starobinsky models with $n=1 . .9$ (from the bottom to the top), as well as GR (direct line) in the logarithmic scale

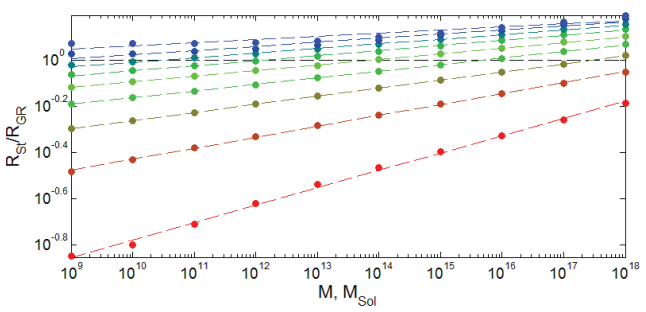

Figure 2. The ratio of the rotation radius of the Starobinsky model to the same value in GR against the mass. Here the points represent the results of numerical calculations; the dashed lines are approximations by linear dependences. For large $n$, nonlinear effects begin to appear.

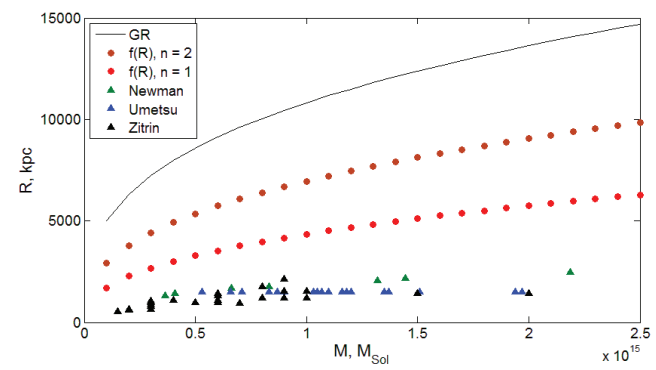

Figure 3. Comparison of the theoretical values of the turning radius with those obtained from observations of masses of clusters. The line denotes the values given by GR. The values for the Starobinsky models with $n=1$ and $n=2$ are denoted by round points, the values for $n=2$ are above. Real data values are indicated by triangles. 


\section{Discussion and Conclusions}

We considered the model of $f(R)$ - gravity with vanishing cosmological constant (the Starobinsky model) on scales of galaxies and their clusters. Turning radius values for masses of galaxy clusters are obtained, as a strict upper bound on the size of the clusters. As a result, it was found that the dependence of the turning radius against the mass of the object for the Starobinsky model differs from that obtained in GR very little. It is established that the turning radius increases with increasing parameter $n$ in all mass range considered. Despite the fact that some models give a much smaller turning radius than GR, no model exceeded the limits set by observational data: $20 \%$ of the radius given by GR.

We can only conclude that the achieved level of accuracy does not allow obtaining additional restrictions on the Starobinsky model with a vanishing cosmological constant on the scales of clusters of galaxies. Thus, at the existing level of accuracy, the Starobinsky model of $f(R)$ gravity, and, consequently, the scalar-tensor models associated with this one by the transformations do not contradict the astronomical data on the scales of galaxies and their clusters. We also want to emphasize the perspectives of the proposed method for testing other extended gravity models.

\section{Acknowledgements}

The work was supported by the RFBR grant 16-02-00682. The authors are grateful to Maxim Pshirkov for interesting discussions and critical remarks.

\section{References}

[1] C.M. Will, Living Rev. Rel. 17, 4 (2014), 1403. 7377

[2] B.P. Abbott et al. (Virgo, LIGO Scientific), Phys. Rev. Lett. 116, 061102 (2016), 1602.03837

[3] B.P. Abbott et al. (Virgo, LIGO Scientific), Phys. Rev. Lett. 116, 221101 (2016), 1602 . 03841

[4] P.A.R. Ade et al. (Planck), Astron. Astrophys. 594, A13 (2016), 1502.01589

[5] Ya.B. Zel'dovich, A. Krasinski, Ya.B. Zeldovich, Sov. Phys. Usp. 11, 381 (1968), [Usp. Fiz. Nauk95,209(1968)]

[6] S. Weinberg, Rev. Mod. Phys. 61, 1 (1989)

[7] M.H. Goroff, A. Sagnotti, Nucl. Phys. B266, 709 (1986)

[8] C.P. Burgess, Living Rev. Rel. 7, 5 (2004), gr-qc/0311082

[9] A.D. Linde, Lect. Notes Phys. 738, 1 (2008), 0705. 0164

[10] S. Capozziello, M. De Laurentis, Phys. Rept. 509, 167 (2011), 1108.6266

[11] T. Clifton, P.G. Ferreira, A. Padilla, C. Skordis, Phys. Rept. 513, 1 (2012), 1106.2476

[12] E. Berti et al., Class. Quant. Grav. 32, 243001 (2015), 1501.07274

[13] A. De Felice, S. Tsujikawa, Living Rev. Rel. 13, 3 (2010), 1002 . 4928

[14] V. Faraoni, Phys. Rev. D72, 124005 (2005), gr-qc/0511094

[15] A.A. Starobinsky, JETP Lett. 86, 157 (2007), 0706. 2041

[16] A.A. Starobinsky, Phys. Lett. B91, 99 (1980)

[17] S. Nojiri, S.D. Odintsov, Phys. Lett. B576, 5 (2003), hep-th/0307071

[18] S. Capozziello, M. Francaviglia, Gen. Rel. Grav. 40, 357 (2008), 0706. 1146

[19] C. Charmousis, Lect. Notes Phys. 892, 25 (2015), 1405. 1612

[20] J. Ntahompagaze, A. Abebe, M. Mbonye, Int. J. Geom. Meth. Mod. Phys. 14, 1750107 (2017), 1706.07722 
[21] R. Nandra, A.N. Lasenby, M.P. Hobson, Mon. Not. Roy. Astron. Soc. 422, 2931 (2012), 1104.4447

[22] V. Faraoni, Phys. Dark Univ. 11, 11 (2016), 1508.00475

[23] J. Kormendy, D.B. Fisher, M.E. Cornell, R. Bender, Astrophys. J. Suppl. 182, 216 (2009), Q810. 1681

[24] B. Binggeli, Astronomy and Astrophysics 107, 338 (1982)

[25] A.I. Zabludoff, M.J. Geller, Astron. J. 107, 1929 (1994)

[26] R.B. et al., IEEE Transactions on Electron Devices 39, 1031 (1983)

[27] M. Fich, S. Tremaine, Ann. Rev. Astron. Astrophys. 29, 409 (1991)

[28] A. Zitrin et al., Astrophys. J. 801, 44 (2015), 1411. 1414

[29] K. Umetsu et al., Astrophys. J. 795, 163 (2014), 1404.1375

[30] A.B. Newman, T. Treu, R.S. Ellis, D.J. Sand, C. Nipoti, J. Richard, E. Jullo, Astrophys. J. 765, 24 (2013), 1209. 1391 